\title{
B3LYP calculations of cerium oxides
}

\author{
Jolla Kullgren, ${ }^{1}$ Christopher W. M. Castleton, ${ }^{2}$ Carsten Müller, ${ }^{1}$ David Muñoz Ramo, ${ }^{3}$ and \\ Kersti Hermansson ${ }^{1, a)}$ \\ ${ }^{1}$ Materials Chemistry, The Angström Laboratory, Uppsala University, Box 538, S-751 21 Uppsala, Sweden \\ ${ }^{2}$ School of Science and Technology, Nottingham Trent University, Nottingham, NG11 8NS, \\ United Kingdom \\ ${ }^{3}$ Department of Physics and Astronomy, University College London and The London Centre for \\ Nanotechnology, Gower Street, London, WC1E 6BT, United Kingdom
}

(Received 10 March 2009; accepted 2 October 2009; published online 5 February 2010)

\begin{abstract}
In this paper we evaluate the performance of density functional theory with the B3LYP functional for calculations on ceria $\left(\mathrm{CeO}_{2}\right)$ and cerium sesquioxide $\left(\mathrm{Ce}_{2} \mathrm{O}_{3}\right)$. We demonstrate that B3LYP is able to describe $\mathrm{CeO}_{2}$ and $\mathrm{Ce}_{2} \mathrm{O}_{3}$ reasonably well. When compared to other functionals, B3LYP performs slightly better than the hybrid functional PBE0 for the electronic properties but slightly worse for the structural properties, although neither performs as well as $L D A+U(U=6 \mathrm{eV})$ or $\mathrm{PBE}+\mathrm{U}(\mathrm{U}=5 \mathrm{eV})$. We also make an extensive comparison of atomic basis sets suitable for periodic calculations of these cerium oxides. Here we conclude that there is currently only one type of cerium basis set available in the literature that is able to give a reasonable description of the electronic structure of both $\mathrm{CeO}_{2}$ and $\mathrm{Ce}_{2} \mathrm{O}_{3}$. These basis sets are based on a 28 electron effective core potential (ECP) and 30 electrons are attributed to the valence space of cerium. Basis sets based on 46 electron ECPs fail for these materials. () 2010 American Institute of Physics.
\end{abstract}

[doi:10.1063/1.3253795]

\section{INTRODUCTION}

Ceria $\left(\mathrm{CeO}_{2}\right)$ is used in car catalysts where it serves as an oxygen buffer and supports the oxidation of carbon monoxide and the decomposition of $\mathrm{NO}_{x}$ and hydrocarbons. Oxygen vacancies in ceria are vital for these processes. Here, quantum mechanical calculations can provide important new insights.

Ceria crystallizes in the fluorite structure $(F m \overline{3} m)$; see Fig. 1(a). A schematic band structure diagram of ceria is presented in Fig. 2(a). The valence band is primarily built from $\mathrm{O} 2 p$ states and the conduction band from $\mathrm{Ce} 5 d$ states. In between these lies a narrow $\mathrm{Ce} 4 f$ band. The $\mathrm{O} 2 p$ $\rightarrow$ Ce $5 d$ band gap has been measured as $6 \mathrm{eV}$ using optical reflectance ${ }^{1}$ and $5.4-8.4 \mathrm{eV}$ using $\mathrm{x}$-ray absorption spectroscopy $(\mathrm{XAS}){ }^{2}$ Bulk excitonic effects were found by Chengyun et $a l .{ }^{3}$ to be on the order of a few meV, so the true band gap is generally thought more likely to lie around $6 \mathrm{eV}^{2,4,5}$ rather than, say, 7 or $8 \mathrm{eV}$. The experimental $\mathrm{O} 2 p \rightarrow \mathrm{Ce} 4 f$ gap has been measured as $2.6 \mathrm{eV}$ using Bremsstrahlung isochromat spectroscopy ${ }^{4}$ (BIS) and 3.33 or $3.39 \mathrm{eV}$ using photoluminescence. ${ }^{6}$

When oxygen vacancies are formed in ceria, the lattice experiences a local distortion and the $\mathrm{Ce} 4 f$ band is split into two bands: an occupied $\mathrm{Ce} 4 f_{\text {Full }}$ band and an empty $\mathrm{Ce}$ $4 f_{\text {Empty }}$ band; see Fig. 2(b). The current understanding is that the occupied $\mathrm{Ce} 4 f$ states are localized atomic $f$-like states at cerium ions neighboring the vacancy. The exact separation of the two $\mathrm{Ce} 4 f$ bands is not clear and there is a rather broad range of values suggested from experiments using $\mathrm{x}$-ray pho-

\footnotetext{
${ }^{a)}$ Electronic mail: kersti@mkem.uu.se.
}

toemission spectroscopy, XAS, BIS, and high resolution electron energy loss spectroscopy. This is due to problems with resolution, line broadening, and also disagreements between different experiments. The uncertainty in the split was recently discussed in some detail by us in Ref. 7. There, we concluded that from existing experiments one can only say that the $\mathrm{Ce} 4 f_{\text {Full }}$ band may lie anywhere in the range $1.5-2.5 \mathrm{eV}$ above the valence band.

The reduction limit of nonstoichiometric ceria is $\mathrm{Ce}_{2} \mathrm{O}_{3}$, where all cerium ions are found in a $\mathrm{Ce}$ (III) oxidation state. The crystal structure of cerium sesquioxide $\left(\mathrm{Ce}_{2} \mathrm{O}_{3}\right)$ under normal conditions is the A-type sesquioxide $(P \overline{3} m 1)$ [Fig. 1(b)]. In the $\mathrm{Ce}_{2} \mathrm{O}_{3}$ band structure we see a resemblance to reduced ceria with the main difference being that the Ce $4 f_{\text {Empty }}$ and Ce $5 d$ bands are merged together in the conduction band; see Fig. 2(c). The experimental Ce $4 f_{\text {Full }}$ $\rightarrow \mathrm{Ce} 4 f / \mathrm{Ce} 5 d$ band gap has been measured as around $2.4 \mathrm{eV}^{8}$

The description of nonstoichiometric ceria and $\mathrm{Ce}_{2} \mathrm{O}_{3}$ proves to be a challenging task for many quantum mechanical (QM) approaches. For example, it is well known that the

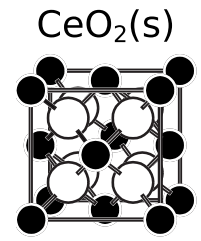

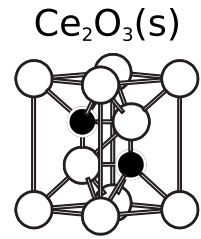

b)

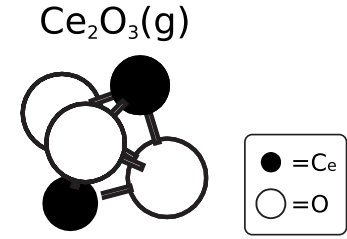

c)
FIG. 1. Three model systems have been used in this study: (a) $\mathrm{CeO}_{2}$ in the fluorite structure $(F m \overline{3} m),\left(\right.$ b) $\mathrm{Ce}_{2} \mathrm{O}_{3}$ in the A-type structure $(P \overline{3} m 1)$, and (c) a one-unit $\mathrm{Ce}_{2} \mathrm{O}_{3}$ cluster. 
standard local density approximation (LDA) and the generalized gradient approximation (GGA) within the density functional theory (DFT) fail to describe the $f$-electron localization properly and the local distortion of the lattice associated with oxygen vacancy formation in ceria. Furthermore, they are not able to give an electronic structure of $\mathrm{Ce}_{2} \mathrm{O}_{3}$ consistent with experiment. It has been shown by several authors ${ }^{7,9-13}$ that the DFT $+\mathrm{U}^{37}$ method can be used to overcome these problems. However, if we wish to study interactions between molecules and the ceria surface using theoretical methods it is desirable to use a method which is known to describe both the bulk material and the molecule in a satisfactory way. Hybrid functionals, and in particular B3LYP, ${ }^{14-17}$ have been used successfully to describe many properties of a large variety of systems, and there is therefore an interest in applying hybrid functionals to cerium oxides. Recently, Hay et al. ${ }^{18}$ performed periodic LDA, the PBE (Perdew-Burke-Ernzerhof ${ }^{19}$ ) variant of GGA, TPSS (Tao-Perdew-Staroverov-Scuseria ${ }^{20}$ ) and HSE (HeydScuseria-Ernzerhof ${ }^{21}$ ) calculations on ceria and $\mathrm{Ce}_{2} \mathrm{O}_{3}$ with atomic basis sets. They concluded that it is possible to obtain a good description of both $\mathrm{CeO}_{2}$ and $\mathrm{Ce}_{2} \mathrm{O}_{3}$, consistent with experiments, by using the hybrid functional HSE. Da Silva et $a l .{ }^{13}$ performed similar calculations using a plane-wave (PW) basis set and the functionals $\mathrm{LDA}, \mathrm{PBE}, \mathrm{LDA}+\mathrm{U}, \mathrm{PBE}+\mathrm{U}$, PBE0, ${ }^{38,39}$ and HSE and reached the same conclusion regarding the performance of the hybrid functionals, PBE0 and HSE.

In the current study we set out to explore the performance of the B3LYP functional for ceria. The motivation is, as hinted above, the fact that B3LYP is well tested for a large set of chemical systems. If we indeed find that B3LYP is successful in describing both $\mathrm{CeO}_{2}$ and $\mathrm{Ce}_{2} \mathrm{O}_{3}$, the extension to more chemically interesting problems should be straightforward. Bredow and Gerson ${ }^{22}$ showed that the B3LYP method is able to give a consistent description of the transition metal oxides, $\mathrm{NiO}$ and $\mathrm{CoO}$, and the ionic oxide $\mathrm{MgO}$. Furthermore, Muscat et al $^{23}$ showed that B3LYP gives a reasonable description of the electronic structure of semiconductors ( $\mathrm{Si}$, diamond and $\mathrm{GaAs})$, semi-ionic oxides $\left(\mathrm{ZnO}, \mathrm{Al}_{2} \mathrm{O}_{3}, \mathrm{TiO}_{2}\right)$, sulphides $\left(\mathrm{FeS}_{2}, \mathrm{ZnS}\right)$, transition metal oxides $(\mathrm{MnO}, \mathrm{NiO})$, and the ionic oxide $\mathrm{MgO}$. Vetere et al. ${ }^{24}$ made a comparison between the B3LYP and PBE0 functionals for a set of $\mathrm{LnX}_{3}$ complexes $(\mathrm{X}=\mathrm{F}, \mathrm{Cl}, \mathrm{Br}, \mathrm{I}$ and $\mathrm{Ln}$ $=\mathrm{La}, \mathrm{Gd}, \mathrm{Lu})$ and concluded that while B3LYP gives very good agreement with experimental data for bond lengths, bond angles, relative energies, and frequencies, PBE0 gives even better agreement. There are only three B3LYP investigations of ceria in the literature, to the best of our knowledge. The two first of these are the embedded-cluster calculations of $\mathrm{CO}$ adsorption on $\mathrm{CeO}_{2}(110)$ by Müller et al. ${ }^{25}$ and Herschend et al. $^{26}$ The third is the study of sulfur interaction with the ceria surface described by Baranek et al. ${ }^{27}$ None of these make a full assessment of the performance of the functional for the cerium oxides.

The basis set is a central part in any QM calculation and in this paragraph we give a background to some of the basis sets described in the literature for cerium. Many QM calculations utilize atomic basis sets based on Gaussian type or-

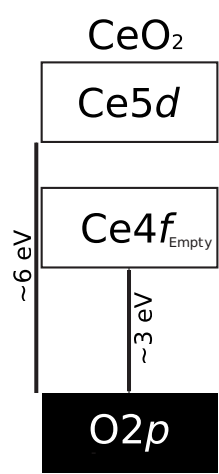

(a)

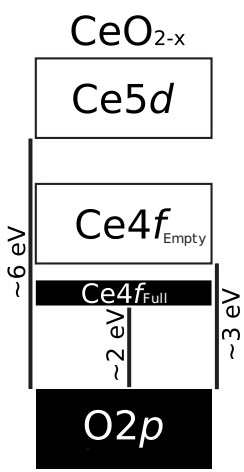

(b)

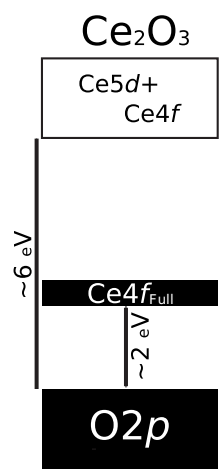

(c)
FIG. 2. A schematic diagram of the band structure of $\mathrm{CeO}_{2}, \mathrm{CeO}_{2-x}$, and $\mathrm{Ce}_{2} \mathrm{O}_{3}$.

bitals. For the heavier elements, atomic basis sets make use of effective core potentials (ECPs), where a fixed number of core electrons are replaced by an effective potential. The total electron configuration of cerium is $[\mathrm{Kr}] 4 d^{10} 5 s^{2} 5 p^{6} 4 f^{1} 5 d^{1} 6 s^{2}$. The Stuttgart-Dresden group (Dolg $e a_{a l}{ }^{28}$ ) have developed ECPs and the corresponding valence basis sets for the rare-earth elements. They found that large-core ECPs with 46 electrons in the core and only the $5 s, 5 p, 4 f, 5 d$, and $6 s$ electrons treated as valence electrons lead to errors in excitation energies (the excitations considered are described in Ref. 29) of several tenths of an $\mathrm{eV}$ compared to a fully variational all-electron (AE) reference. This was not considered to be acceptable, and it was not until they added the $4 s, 4 p$, and $4 d$ electrons to the valence space that they obtained an almost perfect agreement. The cerium ECP of this type (small-core) thus only contains 28 electrons and the remaining 30 electrons are attributed to the valence space. Cao et al. ${ }^{30}$ later derived atomic natural orbital (ANO) and segmented contractions (SEG) (Ref. 31) valence basis sets for this ECP. These basis sets will hereafter be called $\mathrm{Ce}$ (RSCANO) and $\mathrm{Ce}(\mathrm{RSCSEG})$, respectively. Later the Stuttgart/Dresden group published another valence basis set for the same ECP on the EMSL basis set exchange home page. ${ }^{32}$ This basis set is entitled "Stuttgart RSC 1997 ECP" and we will hereafter denote it Ce(RSC97). Dolg et $a l .{ }^{33}$ have also developed a basis set for cerium with a largecore (46-electron) ECP, hereafter called Ce(LC1). Huelsen et $a l .{ }^{34}$ developed augmented triple zeta and augmented quadruple zeta basis sets for cerium using another 46-electron ECP. These two basis sets will be denoted $\mathrm{Ce}(\mathrm{LC} 2)$ and $\mathrm{Ce}(\mathrm{LC} 3)$, respectively. Cundari et al. ${ }^{35}$ constructed another large-core ECP and accompanying valence basis sets for the lanthanide series following a scheme that Stevens et al. ${ }^{36}$ had previously used successfully to construct basis sets for transition metals. In Ref. 35 such ECPs were optimized for the trivalent lanthanide ions. The cerium basis set of this type will hereafter be denoted $\mathrm{Ce}(\mathrm{SBKJC})$.

In this study we present a systematic comparison of the performance of several localized atomic basis sets and DFT functionals, assessing their ability to describe the electronic structure and mechanical properties of $\mathrm{CeO}_{2}$ and $\mathrm{Ce}_{2} \mathrm{O}_{3}$. We will particularly focus on the performance of the B3LYP functional. 
TABLE I. Contraction schemes of basis sets.

\begin{tabular}{lccc}
\hline \hline Label & Ref. & ECP size & Notation $^{\mathrm{a}}$ \\
\hline Ce(RSCANO) & 30 & $28 e^{-}$ & $\mathrm{Ce}(84 s 78 p 50 d 32 f 18 g) \rightarrow[6 s 6 p 5 d 4 f 3 g]$ \\
Ce(RSCSEG) & 31 & $28 e^{-}$ & $\mathrm{Ce}(14 s 13 p 10 d 8 f 6 g) \rightarrow[10 s 8 p 5 d 4 f 3 g]$ \\
Ce(RSC97) & 32 & $28 e^{-}$ & $\mathrm{Ce}(38 s 23 p 11 d 10 f) \rightarrow[5 s 5 p 4 d 3 f]$ \\
$\mathrm{Ce}($ SBKJC) & 35 & $46 e^{-}$ & $\mathrm{Ce}(6 s 6 p 3 d 7 f) \rightarrow[4 s 4 p 2 d 2 f]$ \\
$\mathrm{Ce}(\mathrm{LC} 1)$ & 33 & $46 e^{-}$ & $\mathrm{Ce}(7 s 6 p 5 d 2 f) \rightarrow[5 s 4 p 3 d 2 f]$ \\
$\mathrm{Ce}(\mathrm{LC} 2)$ & 34 & $46 e^{-}$ & $\mathrm{Ce}(7 s 6 p 5 d 2 f) \rightarrow[5 s 4 p 4 d 2 f]$ \\
$\mathrm{Ce}(\mathrm{LC} 3)$ & 34 & $46 e^{-}$ & $\mathrm{Ce}(7 s 6 p 5 d 2 f 1 g) \rightarrow[6 s 5 p 4 d 2 f 1 g]$ \\
$\mathrm{O}(8-51 \mathrm{G})$ & 45 & $\ldots$ & $\mathrm{O}(14 s 6 p) \rightarrow[3 s 2 p]$ \\
$\mathrm{O}(6-31 \mathrm{G})$ & 46 & $\ldots$ & $\mathrm{O}(10 s 4 p) \rightarrow[3 s 2 p]$ \\
$\mathrm{O}\left(6-31 \mathrm{G}^{*}\right)$ & 46 and 47 & $\ldots$ & $\mathrm{O}(10 s 4 p 1 d) \rightarrow[3 s 2 p 1 d]$ \\
$\mathrm{O}(\mathrm{cc}-\mathrm{pVTZ})$ & 48 & $\mathrm{O}(18 s 5 p 2 d) \rightarrow[4 s 3 p 2 d]$ \\
\hline \hline
\end{tabular}

$\overline{\bar{a} \text { In this notation the round (normal) parenthesis refers to an uncontracted basis set and the square brackets to the }}$ corresponding contracted basis set.

\section{METHOD}

\section{A. Model systems}

Three different model systems, two crystals and one cluster, are used in this work. The crystals are the $\mathrm{CeO}_{2}$ fluorite crystal $(F m \overline{3} m)$ and the $\mathrm{Ce}_{2} \mathrm{O}_{3}$ A-type crystal $(P \overline{3} m 1)$. These two structures are depicted in Figs. 1(a) and 1(b). The third model system is a one-formula unit $\mathrm{Ce}_{2} \mathrm{O}_{3}$ cluster, constructed as a trigonal bipyramid with two cerium ions sharing three oxygens in between them [Fig. 1(c)].

\section{B. Computational details}

Five different functionals were used in this study: LDA, $\mathrm{LDA}+\mathrm{U}$, the GGA functional PBE, ${ }^{19}$ PBE0, and B3LYP. ${ }^{14-17}$ The latter two are hybrid functionals and include $25 \%$ and $20 \%$ Hartree-Fock (HF) exchange, respectively. PBE0 is a one-parameter hybrid functional and can be expressed in the following way:

$$
E_{\mathrm{XC}}^{\mathrm{PBE} 0}=E_{\mathrm{XC}}^{\mathrm{PBE}}+\frac{1}{4}\left(E_{X}^{\mathrm{HF}}-E_{X}^{\mathrm{PBE}}\right),
$$

where $E_{\mathrm{XC}}$ denotes exchange-correlation energy and $E_{X}$ denotes exchange energy. B3LYP employs a more elaborated functional with three parameters and has the general form,

$$
\begin{aligned}
E_{\mathrm{XC}}^{\mathrm{B} 3 \mathrm{LYP}}= & E_{\mathrm{XC}}^{\mathrm{LDA}}+a_{0}\left(E_{X}^{\mathrm{HF}}-E_{X}^{\mathrm{LDA}}\right)+a_{X}\left(E_{X}^{\mathrm{GGA}}-E_{X}^{\mathrm{LDA}}\right) \\
& +a_{C}\left(E_{C}^{\mathrm{GGA}}-E_{C}^{\mathrm{LDA}}\right),
\end{aligned}
$$

with parameters $a_{0}=0.20, a_{X}=0.72$, and $a_{C}=0.81$. Here $E_{C}$ denotes correlation energy and $E_{\mathrm{XC}}$ and $E_{X}$ were defined above.

Three different codes were used: GAUSSIAN03 (Ref. 40) for the local basis set calculation of the $\mathrm{Ce}_{2} \mathrm{O}_{3}$ cluster, CRYSTAL06 (Ref. 41) for the local basis set calculations of the crystals and the Vienna $A b$ initio Simulation Package (VASP) ${ }^{42,43}$ for PW reference calculations. The GAUSSIAN03 and CRYSTAL06 programs both use atomic basis sets while VASP uses PW basis sets. All GAUSSIAN03 calculations were carried out using a tight convergence criterion. In the periodic calculations with CRYSTAL06 we sampled the Brillouin zone using a $10 \times 10 \times 10$ Monkhorst-Pack ${ }^{44}(\mathrm{MP})$ grid and in the periodic calculations with VASP we sampled the Brillouin zone with a $10 \times 10 \times 10 \mathrm{MP}$ grid for $\mathrm{Ce}_{2} \mathrm{O}_{3}$ and a $16 \times 16 \times 16 \mathrm{MP}$ grid for $\mathrm{CeO}_{2}$. In the CRYSTAL06 calculations the parameters controlling the threshold for the Coulomb and exchange integrals were set to $(777725)$, the threshold for integrals involving the ECP was set to $10^{-8}$ and the threshold for energy convergence was set to $10^{-7}\left(10^{-10}\right.$ in the calculation of elastic properties). In the VASP calculations, the convergence criterion for the electronic and ionic updates were set to $10^{-7}$ and $10^{-5} \mathrm{eV}$, respectively. The PW reference calculations for the $\mathrm{Ce}_{2} \mathrm{O}_{3}$ cluster were performed using a $10 \times 10 \times 10 \AA^{3}$ cell.

\section{Basis sets}

The Ce basis sets used in this study can be divided into two main groups according to the number of electrons frozen in the ECP. The first group consists of the Stuttgart/Dresden basis sets Ce(RSCANO), Ce(RSCSEG), and Ce(RSC97), all using the same 28-electron ECP, as explained in Sec. I. The second group of basis sets consists of the $\mathrm{Ce}(\mathrm{SBKJC})$, $\mathrm{Ce}(\mathrm{LC} 1), \mathrm{Ce}(\mathrm{LC} 2)$, and $\mathrm{Ce}(\mathrm{LC} 3)$ basis sets, which all use a 46-electron ECP. The two groups of basis sets will be referred to as "small-core" and "large-core" basis sets, respectively. Information about the number of basis functions and primitive Gaussians as well as the number of electrons treated by the ECP is summarized in Table I. Furthermore, in order to perform efficient periodic calculations for $\mathrm{CeO}_{2}$ and $\mathrm{Ce}_{2} \mathrm{O}_{3}$ we have here explored various modified and truncated versions of the $\mathrm{Ce}$ (RCS97) basis set. Three such basis sets were used in this study. The first of these, hereafter called $\mathrm{Ce}$ (RSC97-Tr.1), is essentially the basis set described by Hay et $a l .{ }^{18}$ except that we also removed the $g$-projector from the ECP. Hay et al. ${ }^{18}$ obtained their basis set from the original $\mathrm{Ce}$ (RSC97) basis set by removing all $g$-functions, truncating the basis set by removing the most diffuse basis functions of the $s^{-}, p-$, and $d$-shells, respectively, and further removing two Gaussian type orbitals, those with the smallest exponent, from each contraction. Additionally, the exponent of the most diffuse function left in each shell was set to 0.15 . This basis set then had the following contraction scheme (the key to our notation is given as a footnote in Table I): $\mathrm{Ce}(31 s 18 p 8 d 8 f)$ 
TABLE II. Contraction schemes of the truncated basis sets used in the periodic calculations.

\begin{tabular}{lc}
\hline \hline Abbrev. & \multicolumn{1}{c}{ Notation $^{\mathrm{a}}$} \\
\hline $\mathrm{Ce}($ RSC97-Tr.1) & $\mathrm{Ce}(31 s 18 p 8 d 8 f) \rightarrow[4 s 4 p 3 d 3 f]$ \\
$\mathrm{Ce}($ RSC97-Tr.2) & $\mathrm{Ce}(31 s 18 p 8 d 8 f) \rightarrow[4 s 4 p 3 d 3 f]$ \\
$\mathrm{Ce}($ RSC97-Tr.3) & $\mathrm{Ce}(20 s 18 p 8 d 7 f) \rightarrow[2 s 4 p 3 d 2 f]$ \\
\hline \hline
\end{tabular}

${ }^{\text {a See footnote of Table I. }}$

$\rightarrow[4 s 4 p 3 d \mathrm{ff}]$. We also introduce a more simple truncation of the $\mathrm{Ce}(\mathrm{RSC} 97)$, denoted $\mathrm{Ce}(\mathrm{RSC} 97-\mathrm{Tr} .2)$, which is derived in the same way as the Ce(RSC97-Tr.1) except that we do not adjust any exponents. Finally, a third basis set was developed from the Ce(RSC97-Tr.1) in order to solve inconsistencies with some of the results, as described in Sec. III. This basis set is hereafter denoted Ce(RSC97-Tr.3). The details of this basis set are given in Sec. III. Information about the truncated basis sets are summarized in Table II.

For oxygen we use four different basis sets: $\mathrm{O}(8-51 \mathrm{G}),{ }^{45}$ $\mathrm{O}(6-31 \mathrm{G}),{ }^{46} \mathrm{O}\left(6-31 \mathrm{G}^{*}\right),{ }^{46,47}$ and $\mathrm{O}(\mathrm{cc}-\mathrm{pVTZ}) .{ }^{48}$ The latter two include polarization functions of $d$-type while the first two do not. We choose not to include the $f$-function in the $\mathrm{O}(\mathrm{cc}-\mathrm{pVTZ})$ basis set.

As a reference basis set, for comparison, we have chosen a well converged PW basis set with a cutoff of $500 \mathrm{eV}$ (36.7 Ry). In this basis set, 12 electrons are attributed to the valence space of cerium and six electrons are attributed to the valence space of oxygen. The remaining electrons are treated as core electrons using the projector augmented wave (PAW) method of Blöchl ${ }^{49}$ as implemented in the VASP code. The PAW potential used here were chosen from several alternative PAW potentials supplied with VASP for $\mathrm{Ce}$ and $\mathrm{O}$, including soft $\left(\mathrm{Ce}^{s}\right.$ and $\left.\mathrm{O}^{s}\right)$ and standard $(\mathrm{Ce}$ and $\mathrm{O})$ potentials. For defect-free ceria, the $\mathrm{CeO}$ and $\mathrm{CeO}^{s}$ combinations give almost identical lattice parameters, bulk moduli, and $\mathrm{O} 2 p \rightarrow \mathrm{Ce} 4 f_{\text {Empty }}$ and $\mathrm{O} 2 p \rightarrow \mathrm{Ce} 5 d$ gaps. The $\mathrm{Ce}^{s} \mathrm{O}$ and $\mathrm{Ce}^{s} \mathrm{O}^{s}$ combinations fail however, due to the appearance of a ghost state: an empty band of cerium $s$ character, $0.4 \mathrm{eV}$ above the valence band (hence below the Ce $4 f_{\text {Empty }}$ band). Such ghost states have been seen to occur for certain combinations of local-potentials and nonlocal projectors. ${ }^{50} \mathrm{We}$, therefore, selected the $\mathrm{CeO}^{s}$ combination.

\section{RESULTS AND DISCUSSION}

Here we will report how the various basis sets and functionals presented above perform in calculations for ceria, both stoichiometric and nonstoichiometric, and $\mathrm{Ce}_{2} \mathrm{O}_{3}$. In
Sec. III B we will discuss the performance of the localized basis sets based on results from calculations using the one formula unit $\mathrm{Ce}_{2} \mathrm{O}_{3}$ cluster [Fig. 1(c)]. Prior to that we discuss in Sec. III A some features of our probe system, the one-formula-unit $\mathrm{Ce}_{2} \mathrm{O}_{3}$ cluster. The performance of functionals and basis sets in periodic calculations for $\mathrm{CeO}_{2}$ and $\mathrm{Ce}_{2} \mathrm{O}_{3}$ crystals are discussed in Secs. III C-III F. Our focus will be on the B3LYP functional.

\section{A. The $\mathrm{Ce}_{2} \mathrm{O}_{3}$ cluster-LDA reference calculations}

The small one-unit cluster [Fig. 1(c)] was optimized using the LDA functional and the $\mathrm{Ce}(\mathrm{SBKJC}) / \mathrm{O}(8-51 \mathrm{G})$ basis set and was kept in this structure in all of the calculations. In this cluster the $\mathrm{Ce}-\mathrm{Ce}$ distance is $2.96 \AA$ and the $\mathrm{Ce}-\mathrm{O}$ distances are 2.14-2.17 $\AA$. The resolution in all density of states (DOS) plots presented here is $0.1 \mathrm{eV}$. The $f$-projection in the DOS is of Mulliken type for the atomic basis sets and of Wigner-Seitz type for the PW basis sets.

The LDA functional does not give a correct description of the band structure of $\mathrm{Ce}_{2} \mathrm{O}_{3}$, but at this point we are only interested in whether the cluster can serve as a useful probe for finding basis sets suitable for bulk $\mathrm{Ce}_{2} \mathrm{O}_{3}$. The electronic DOS for both the $\mathrm{Ce}_{2} \mathrm{O}_{3}$ cluster and the $\mathrm{Ce}_{2} \mathrm{O}_{3}$ bulk system is presented in Fig. 3. Naturally, we do expect some differences in the DOS diagrams of the two systems. Indeed, we obtain a difference in the separation of the $\mathrm{Ce} 5 d$ and $\mathrm{Ce}$ $4 f_{\text {Empty }}$ bands, which is more pronounced in the cluster, but apart from this feature, the resemblance is quite striking. This in itself is not sufficient evidence to determine whether the cluster is a useful probe. However, in Sec. III C we will find that there is also a close correspondence between the $\mathrm{Ce}_{2} \mathrm{O}_{3}$ test cluster and the $\mathrm{Ce}_{2} \mathrm{O}_{3}$ crystal with respect to failures in the DOS description of the electronic structure for certain basis sets. The $\mathrm{Ce}_{2} \mathrm{O}_{3}$ cluster tests, presented in Sec. III B and in Figs. 3-6, are therefore also relevant as a screening tool in the selection of suitable basis sets for bulk $\mathrm{Ce}_{2} \mathrm{O}_{3}$.

\section{B. Comparison of large-core and small-core basis sets for cerium using the $\mathrm{Ce}_{2} \mathrm{O}_{3}$ cluster}

Using the $\mathrm{Ce}_{2} \mathrm{O}_{3}$ test cluster, the performance of the large-core and small-core basis sets was evaluated by comparing their DOS diagrams with the DOS calculated using the converged PW basis set. Here we used the LDA functional. Again, the failures of LDA are irrelevant at this stage since we simply want to find out which basis sets are able to reproduce the DOS diagrams of the PW reference.
DOS of $\mathrm{Ce}_{2} \mathrm{O}_{3}(\mathrm{~s})$ and $\mathrm{Ce}_{2} \mathrm{O}_{3}(\mathrm{~g})$, LDA

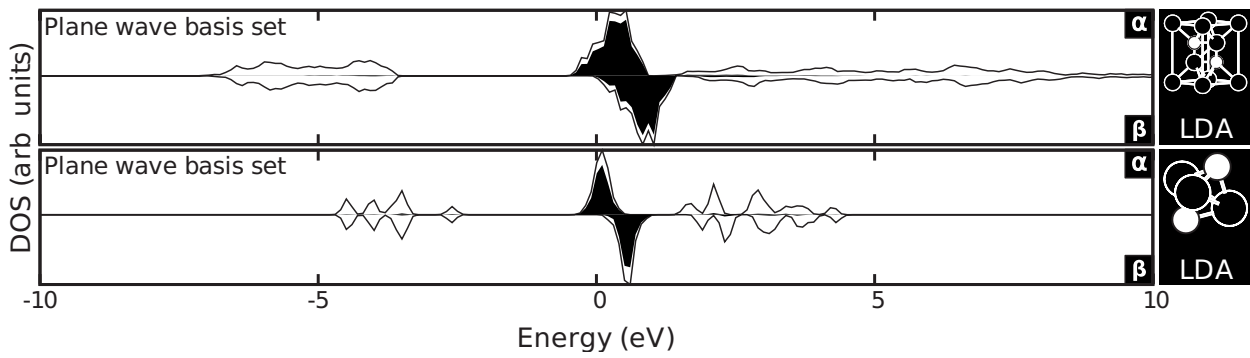

FIG. 3. DOS of the $\mathrm{Ce}_{2} \mathrm{O}_{3}$ crystal and $\mathrm{Ce}_{2} \mathrm{O}_{3}$ cluster using LDA and a PW basis set. Here and in the following figures, a solid line indicates the total DOS while the filled curve shows the $f$-projected DOS. The zero energy corresponds to the energy of the highest occupied band or orbital. Note that the systems and the functionals are shown in the small figures to the right. 
Comparison of large-core cerium basis sets

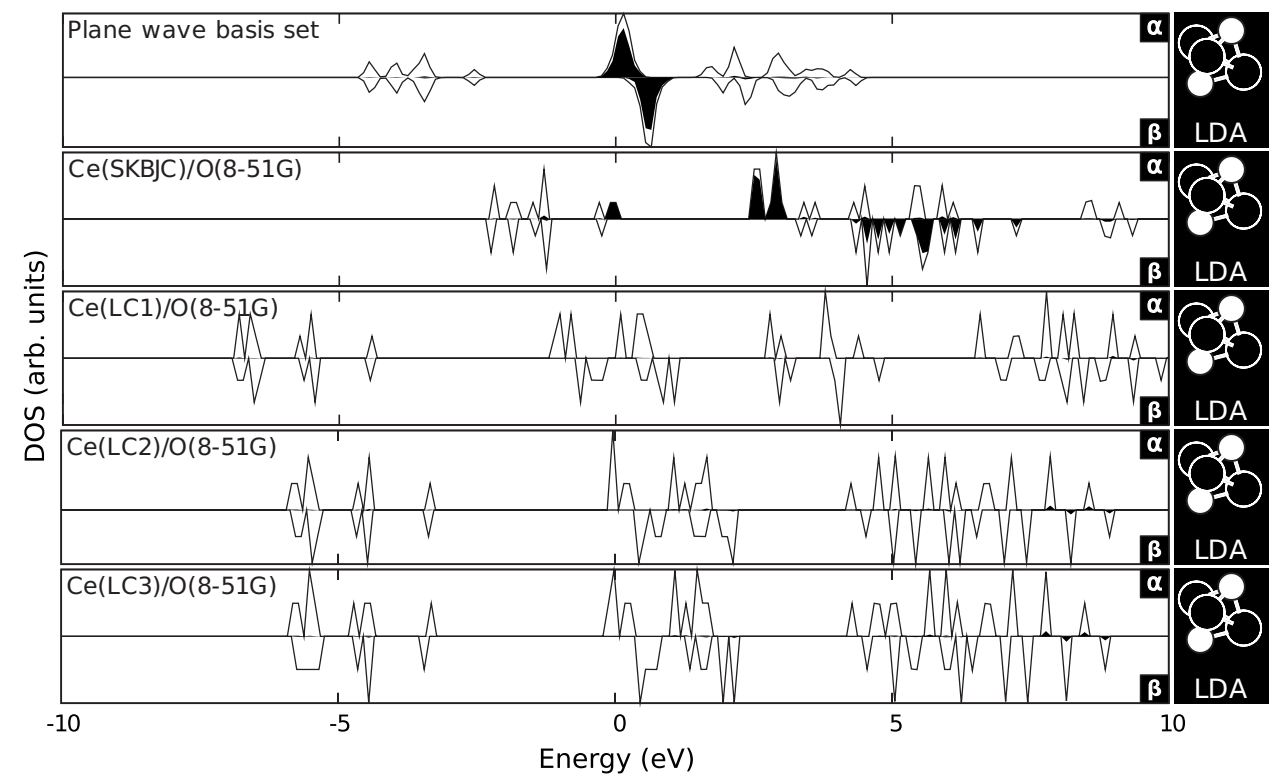

FIG. 4. DOS for the one-unit $\mathrm{Ce}_{2} \mathrm{O}_{3}$ cluster in the triplet state calculated using the large-core basis sets, $\mathrm{Ce}(\mathrm{S}$ BKJC), $\mathrm{Ce}(\mathrm{LC} 1), \quad \mathrm{Ce}(\mathrm{LC} 2), \quad$ and $\mathrm{Ce}(\mathrm{LC} 3)$, for cerium in combination with the $8-51 \mathrm{G}$ basis set for oxygen. The functional used is LDA.
If we first devote our attention to the basis sets with an ECP containing 46 electrons, we notice large discrepancies in the DOS results (Fig. 4). The DOSs from these different atomic basis sets do not agree with each other, neither does any of them reproduce the PW reference. Another version of $\mathrm{Ce}(\mathrm{SBKJC})$ was also constructed in which all contractions were broken up. Even with this rather large basis set there was still no significant improvement in the DOS.

On the other hand, the small-core basis sets, with only 28 electrons in the ECP display large uniformity and they all compare well with the PW reference (Fig. 5). In Fig. 6 we compare the DOS for the $\mathrm{Ce}_{2} \mathrm{O}_{3}$ cluster calculated using the truncated basis sets to the results with the original $\mathrm{Ce}(\mathrm{RSC} 97)$ basis set. We see only small changes in the DOS diagrams.

We also examined the influence of the oxygen basis set (8-51G, 6-31G, 6-31G*, and cc-pVTZ) for Ce(RSC97) and $\mathrm{Ce}(\mathrm{RSC} 97-\mathrm{Tr} .1)$. We find only very minor differences in the DOS figures (not shown here).

We conclude that the large-core basis sets tested fail to reproduce the PW result while all of the small-core basis sets tested reproduce the PW result well. The qualitative results are independent of the oxygen basis sets examined here. We now go on to examine whether these findings also hold true for bulk $\mathrm{Ce}_{2} \mathrm{O}_{3}$.

\section{Electronic structure of bulk $\mathrm{Ce}_{2} \mathrm{O}_{3}$}

We calculated the DOS of bulk $\mathrm{Ce}_{2} \mathrm{O}_{3}$ in the ferromagnetic configuration using LDA and the large-core cerium basis sets $\mathrm{Ce}(\mathrm{SBKJC}), \mathrm{Ce}(\mathrm{LC} 1), \mathrm{Ce}(\mathrm{LC} 2)$, and $\mathrm{Ce}(\mathrm{LC} 3)$. Necessary adjustments, namely the removal of the most diffuse functions in these basis sets, were made prior to these calculations. Again, just as with the test cluster, none of the basis sets was able to reproduce the PW reference (not shown here).

Next we turn to the small-core cerium basis sets. For $\mathrm{Ce}_{2} \mathrm{O}_{3}$, in the ferromagnetic configuration, the DOS diagrams from LDA calculated using the $\mathrm{Ce}(\mathrm{RSC} 97-\mathrm{Tr}-1)$ and $\mathrm{Ce}$ (RSC97-Tr.2) cerium basis sets in combination with the

\section{Comparison of small-core cerium basis sets}

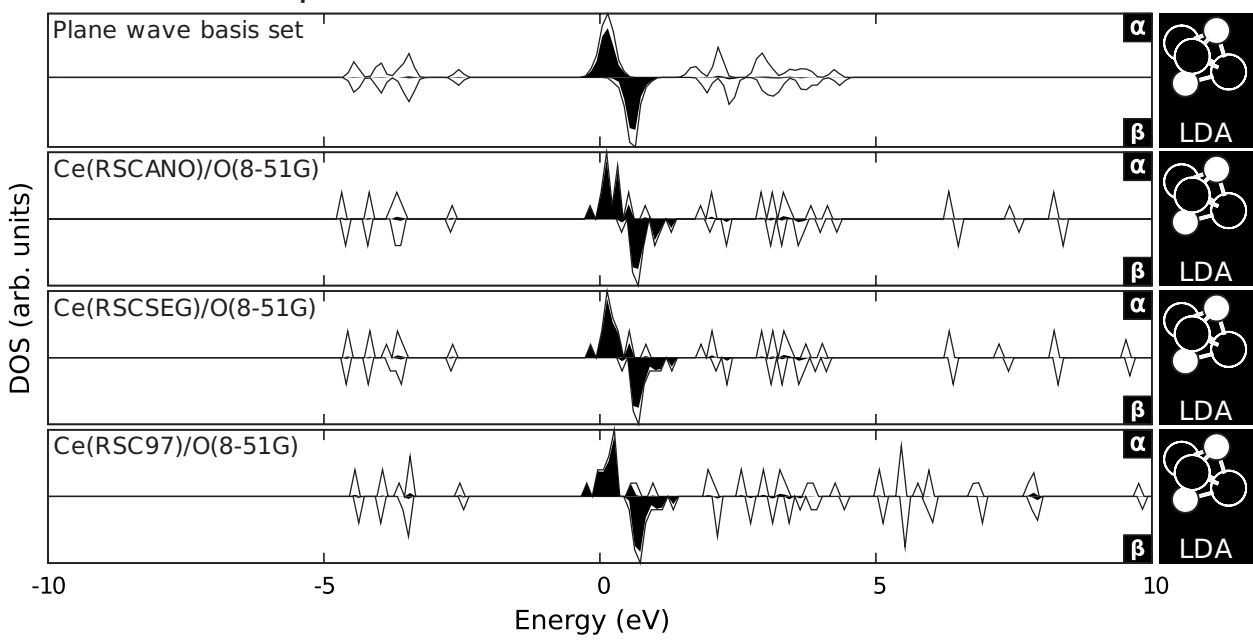

FIG. 5. DOS for the one-unit $\mathrm{Ce}_{2} \mathrm{O}_{3}$ cluster in the triplet state calculated using the small-core basis sets, $\mathrm{Ce}(\mathrm{R}$ SCANO), Ce(RSCSEG), and $\mathrm{Ce}(\mathrm{RSC} 97)$, for cerium in combination with the $\mathrm{O}(8-51 \mathrm{G})$ basis set for oxygen. The functional used is LDA. 
Investigation of truncated small-core cerium basis sets

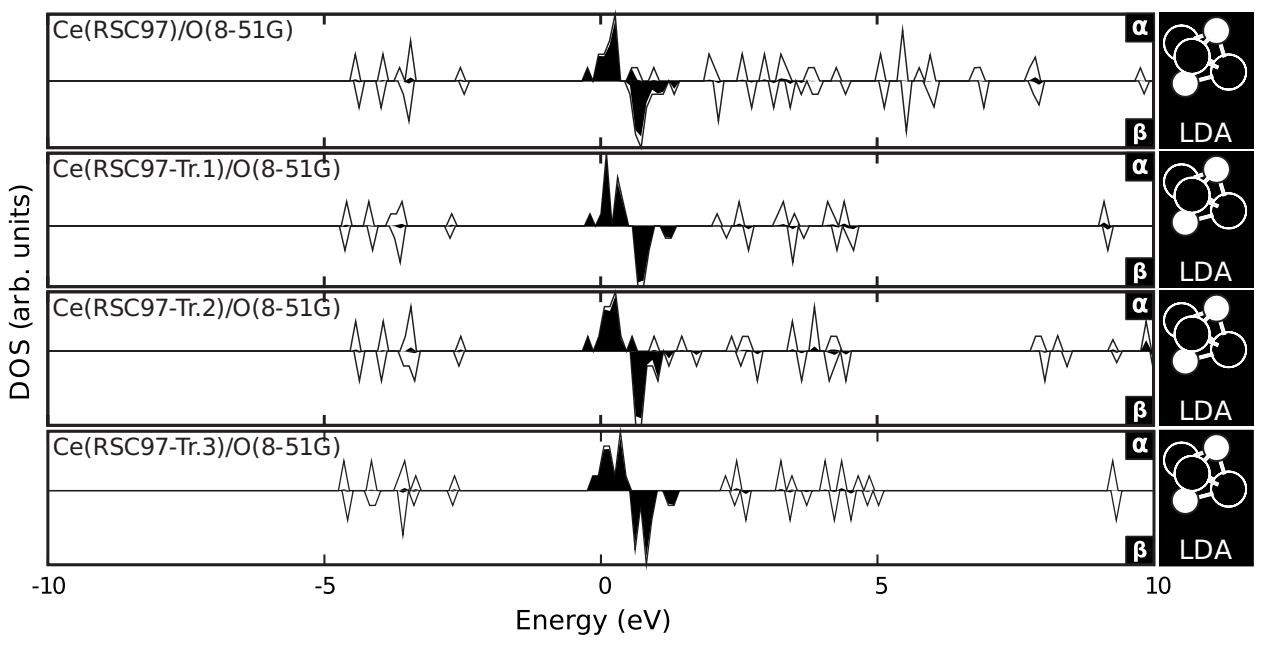

FIG. 6. DOS for the one-unit $\mathrm{Ce}_{2} \mathrm{O}_{3}$ cluster in the triplet state calculated using the truncated $\mathrm{Ce}(\mathrm{RSC} 97)$ cerium basis sets. The functional used is LDA. four oxygen basis sets are presented in Fig. 7. The effect of the oxygen basis set is seen to be quite large, with the larger oxygen basis sets $\mathrm{O}\left(6-31 \mathrm{G}^{*}\right)$ and $\mathrm{O}(\mathrm{cc}-\mathrm{pVTZ})$ promoting a split of the Ce $4 f$ states. In the PBE results (Fig. 8) this effect is even stronger and for the $\mathrm{O}(\mathrm{cc}-\mathrm{pVTZ})$ basis we see a small gap in the DOS. Hay et al. ${ }^{18}$ reported a similar Ce $4 f_{\text {full }}$ $\rightarrow$ Ce $4 f / \mathrm{Ce} 5 d$ gap of $0.3 \mathrm{eV}$ for the antiferromagnetic configuration of $\mathrm{Ce}_{2} \mathrm{O}_{3}$ using $\mathrm{PBE}$ and their truncation of the
$\mathrm{Ce}(\mathrm{RSC} 97)$ basis set together with the $\mathrm{O}\left(6-31 \mathrm{G}^{*}\right)$ basis set. All of these results contradict the PAW-based PW results presented in Fig. 7, where no splitting is observed using PBE. Nevertheless, Fabris et al. ${ }^{10}$ earlier found similar gaps in their ultrasoft pseudopotential (US-PP) based PW calculations using LDA. However, Kresse et al. ${ }^{51}$ showed that this was a result of their use of US-PPs, and that no such gaps are present in AE calculations. Kresse et al. ${ }^{51}$ also showed that

\section{Investigations of small-core $\mathrm{Ce}$ and various \\ $\mathrm{O}$ basis sets for $\mathrm{Ce}_{2} \mathrm{O}_{3}(\mathrm{~s})$, LDA}

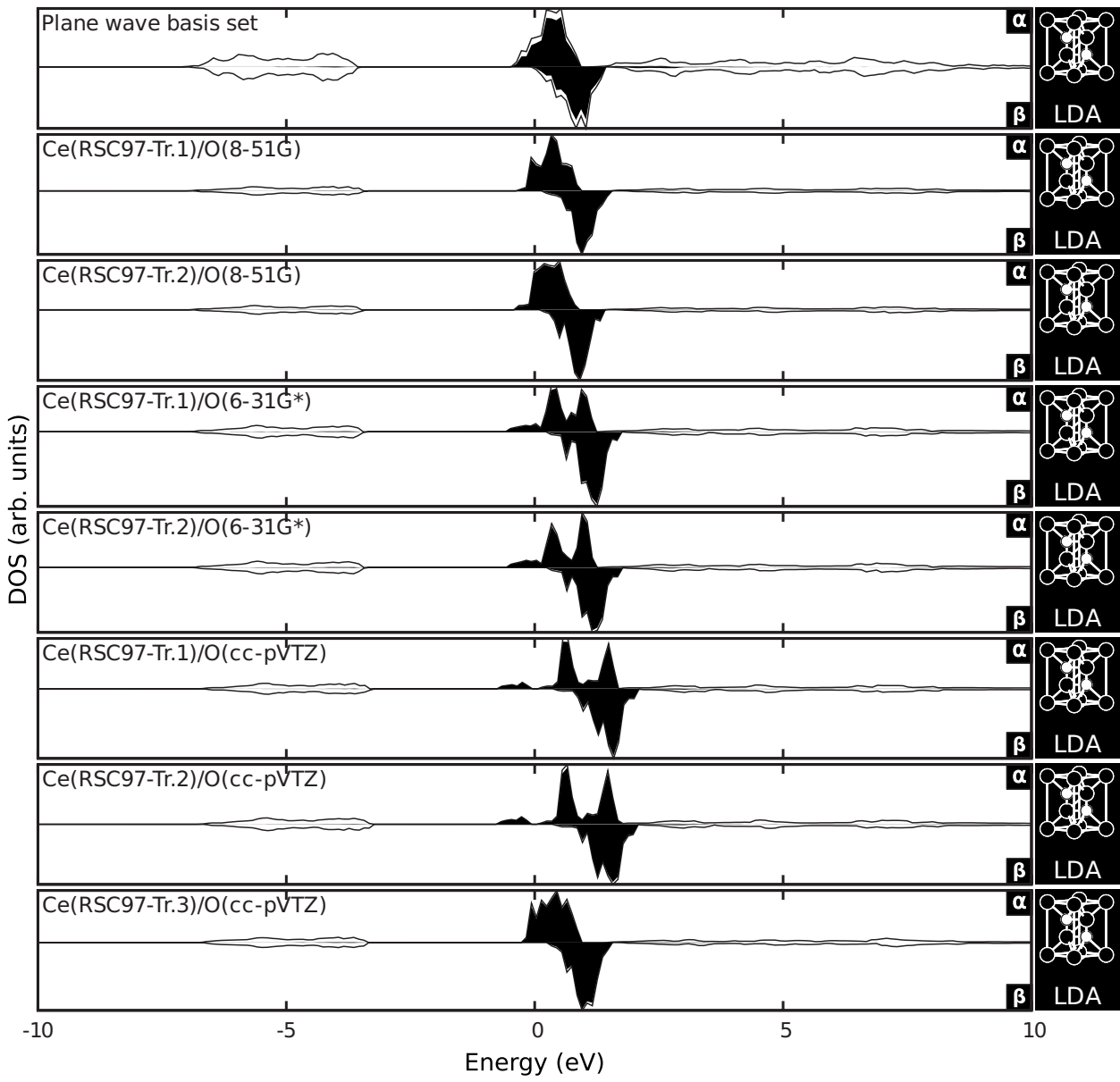

FIG. 7. DOS of bulk $\mathrm{Ce}_{2} \mathrm{O}_{3}$ using the truncated $\mathrm{Ce}(\mathrm{RSC} 97)$ cerium basis sets. Several combinations with different oxygen basis sets are shown. The functional used is LDA. 


\section{Investigations of small-core $\mathrm{Ce}$ and various} $\mathrm{O}$ basis sets for $\mathrm{Ce}_{2} \mathrm{O}_{3}(\mathrm{~s}), \mathrm{PBE}$

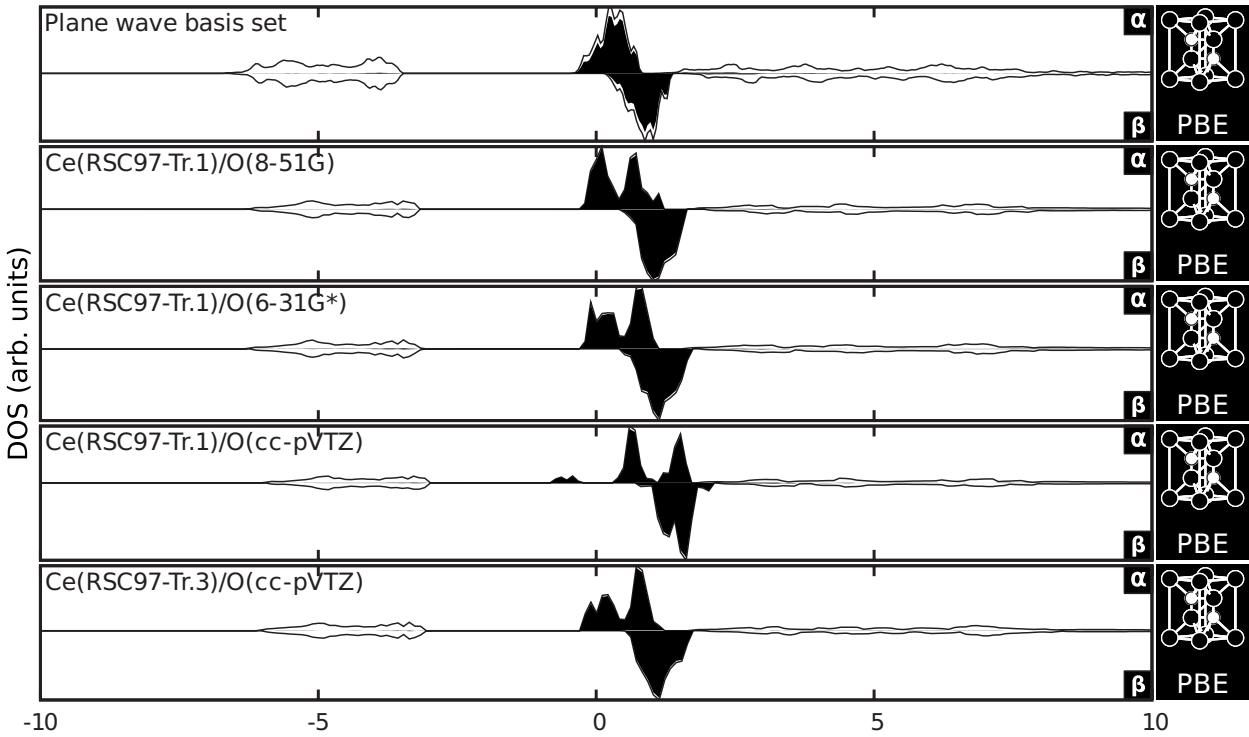

FIG. 8. DOS of bulk $\mathrm{Ce}_{2} \mathrm{O}_{3}$ using two of the truncated $\mathrm{Ce}$ (RSC97) basis sets. Several combinations with different oxygen basis sets are shown. The functional used is PBE. the intermediate PAW method reproduces the AE result rather well, with no gaps. Fabris et al. ${ }^{52}$ addressed the issues raised and agreed that the gaps were indeed due to the USPPs. We find some quantitative differences between the PAW and the AE results, but these are small, certainly much smaller than the differences we find between the PW basis set and even the best of the atomic basis sets. We therefore consider that our PAW potential results provide a valid and sufficiently reliable reference for the type of qualitative comparisons made in the rest of this paper. Hence, we consider the basis sets which lead to the splits in Fig. 7 and in the work of Hay et al., to have failed.

We also note that in the original work by Hay et al. there is a $g$-projector in the ECP which is not included here. This is not expected to make a large difference since the basis set itself does not include any $g$-functions. (Unfortunately, there is no way to test this hypothesis in CRYSTAL06 since this software does not handle $g$-functions or $g$-projectors.)

By replacing the two diffuse $f$-functions $(\xi=0.15$ and $\xi$ $=0.381331)$ with one $f$-function $(\xi=0.3)$ in the Ce(RSC97Tr.1) basis set, the splitting of the $f$-band in the PBE calculations using the $\mathrm{O}(\mathrm{cc}-\mathrm{pVTZ})$ basis set for oxygen is no longer present. We therefore went on to make careful adjust- ments of the diffuse functions in the Ce(RSC97-Tr.1) basis set. In our approach we analyzed the effect of the exponents on both the cell parameters and the electronic structure of $\mathrm{Ce}_{2} \mathrm{O}_{3}$ and searched for a setting that gives a balanced description of both of them. Here we find that the Ce(RSC97Tr.1) basis set with the modification of the $f$-functions just mentioned and with the diffuse $s$-function removed gives a balanced description of both electronic and mechanical properties. Here we used the $\mathrm{O}(\mathrm{cc}-\mathrm{pVTZ})$ oxygen basis in order to treat the cerium and oxygen more consistently. After analyzing the Mulliken population in both $\mathrm{CeO}_{2}$ and $\mathrm{Ce}_{2} \mathrm{O}_{3}$ we found that only two $s$-functions are actually used. We therefore chose to remove the remaining $s$-function from the basis set. This basis set, as mentioned in Sec. II, is denoted $\mathrm{Ce}(\mathrm{RSC}$ 97-Tr.3) and has the following contraction scheme: $\mathrm{Ce}(20 s 18 p 8 d 7 f) \rightarrow[2 s 4 p 3 d 2 f]$.

The LDA and PBE DOS using this basis set are shown in the bottom panels in Figs. 7 and 8. Using the same basis set and the two hybrid functionals PBE0 and B3LYP we obtain the DOSs presented in Fig. 9. Both functionals predict a large separation of the Ce $4 f_{\text {Full }}$ and the conduction bands. The large separation is in qualitative agreement with experimental results ${ }^{8}$ and with literature results for HSE, ${ }^{13,18}$ and

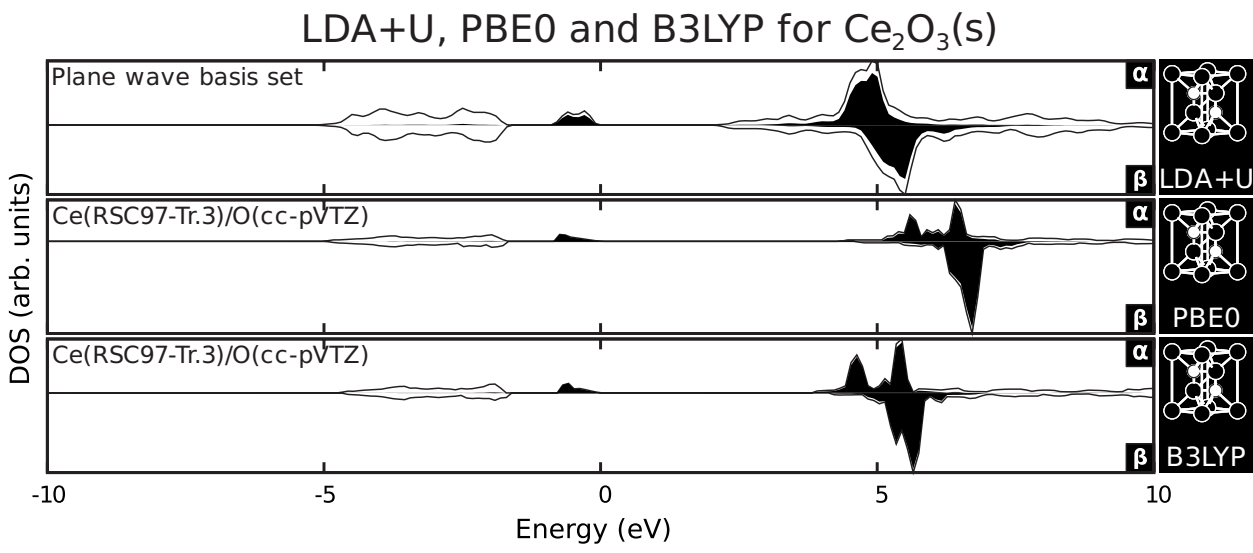

FIG. 9. DOS of bulk $\mathrm{Ce}_{2} \mathrm{O}_{3}$ calculated using $\mathrm{LDA}+\mathrm{U}, \mathrm{PBE} 0$, and B3LYP. In the $\mathrm{LDA}+\mathrm{U}$ calculation a $\mathrm{PW}$ basis set was used and in the PBE0 and B3LYP calculations the Ce(RSC97Tr.3)/O(cc-pVTZ) basis sets were used. 


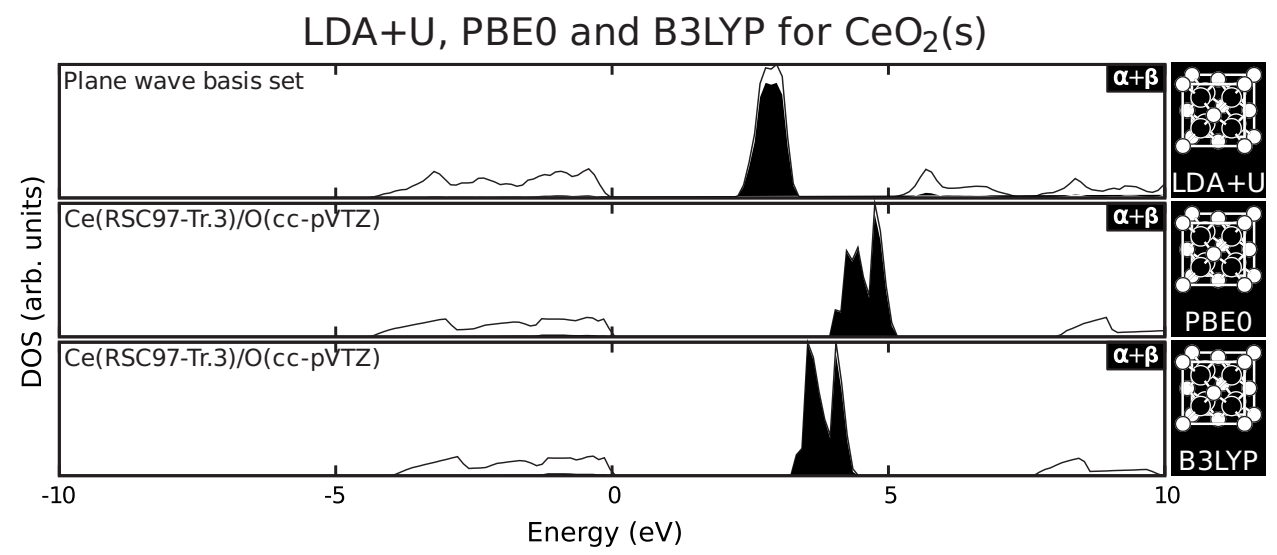

FIG. 10. DOS of bulk $\mathrm{CeO}_{2}$ calculated using LDA+U, PBE0, and B3LYP. In the $\mathrm{LDA}+\mathrm{U}$ calculation a $\mathrm{PW}$ basis set was used and in the PBE0 and B3LYP calculations the Ce(RSC97Tr.3)/O(cc-pVTZ) basis set was used.
$\mathrm{DFT}+\mathrm{U}^{10,11,13}$ functionals. When we compare in more detail the DOS derived from B3LYP and PBE0 to the DOS derived from $\mathrm{LDA}+\mathrm{U}(\mathrm{U}=6 \mathrm{eV})$ using the PW basis set (Fig. 9), we find that the band gap is much larger in the hybrid calculations. The experimental values for the gaps are not sufficiently well defined to judge which of the results is the most accurate.

\section{Electronic structure of bulk $\mathrm{CeO}_{2}$}

In Fig. 10 we present the DOS for bulk $\mathrm{CeO}_{2}$ calculated using the Ce(RSC97-Tr.3)/O(cc-pVTZ) basis set and the PBE0 and B3LYP functionals. We include the PW results for the $\mathrm{LDA}+\mathrm{U}(\mathrm{U}=6 \mathrm{eV})$ functional for comparison. Meanwhile, in Table III we present direct and indirect band gaps of $\mathrm{CeO}_{2}$ calculated with LDA, PBE, PBE0, and B3LYP. Here the $\mathrm{Ce}(\mathrm{RSC} 97-\mathrm{Tr} .1) / \mathrm{O}\left(6-31 \mathrm{G}^{*}\right)$ and $\mathrm{Ce}(\mathrm{RSC} 97-\mathrm{Tr} .3) / \mathrm{O}(\mathrm{cc}-$ pVTZ) basis sets are used. For comparison we also include our PW results for $\mathrm{LDA}+\mathrm{U}$ and $\mathrm{PBE}+\mathrm{U}$ with $\mathrm{U}=6 \mathrm{eV}$ and $\mathrm{U}=5 \mathrm{eV}$, respectively. There are only minor differences between results obtained with the two basis sets while there are large differences in results obtained with the different functionals. Generally, the $\mathrm{O} 2 p \rightarrow \mathrm{Ce} 5 d$ band gaps obtained using the converged PW basis set are smaller than those from the atomic basis set description, while the opposite is true for the $\mathrm{O} 2 p \rightarrow \mathrm{Ce} 4 f_{\text {Empty }}$ band gaps. This behavior is common and may be attributed to a better description of the conduction band using the PW basis set. Atomic basis sets may have limited variational freedom to describe the situations in which significant charge density lies in the interstitial regions, as is common for conduction band states. PW basis sets do not have this problem, although they sometimes struggle with the core region.

The LDA and PBE gaps are much smaller than those obtained using the hybrid functionals, while the LDA $+\mathrm{U}$ and $\mathrm{PBE}+\mathrm{U}$ gaps are intermediate for $\mathrm{O} 2 p \rightarrow \mathrm{Ce} 4 f_{\text {Empty }}$ and the smallest for $\mathrm{O} 2 p \rightarrow \mathrm{Ce} 5 d$. LDA and GGA give the most reasonable values for the $\mathrm{O} 2 p \rightarrow \mathrm{Ce} 5 d$ gaps (here we compare with the $6 \mathrm{eV}$ value suggested in most experimental papers), while their $\mathrm{O} 2 p \rightarrow \mathrm{Ce} 4 f_{\text {Empty }}$ gaps are much smaller than suggested by experimental measurements.

TABLE III. Direct and indirect $\mathrm{CeO}_{2}$ band gaps calculated using the $\mathrm{Ce}(\mathrm{RSC} 97-\mathrm{Tr} .1) / \mathrm{O}\left(6-31 \mathrm{G}^{*}\right)$ and $\mathrm{Ce}$ (RSC97-Tr.3)/O(cc-pVTZ) basis sets compared to PW results using LDA+U and PBE+U, and to experiment.

\begin{tabular}{|c|c|c|c|c|c|}
\hline \multirow[b]{2}{*}{ Functional } & \multirow[b]{2}{*}{ Basis set } & \multicolumn{2}{|c|}{$\begin{array}{c}\mathrm{O} 2 p \rightarrow \mathrm{Ce} 5 d \\
(\mathrm{eV})\end{array}$} & \multicolumn{2}{|c|}{$\begin{array}{c}\mathrm{O} 2 p \rightarrow \mathrm{Ce} \\
(\mathrm{eV})\end{array}$} \\
\hline & & Direct & Indirect & Direct & Indirect \\
\hline LDA & $\mathrm{Ce}(\mathrm{RSC} 97-\mathrm{Tr} .1) / \mathrm{O}\left(6-31 \mathrm{G}^{*}\right)$ & 6.33 & 5.85 & 1.97 & 1.87 \\
\hline LDA & Ce(RSC97-Tr.3)/O(cc-pVTZ) & 6.42 & 5.97 & 1.91 & 1.83 \\
\hline LDA & PW & 6.10 & 5.58 & 2.03 & 1.94 \\
\hline PBE & Ce(RSC97-Tr.1)/O(6-31G*) & 6.36 & 5.93 & 1.79 & 1.72 \\
\hline PBE & $\mathrm{Ce}(\mathrm{RSC} 97-\mathrm{Tr} .3) / \mathrm{O}(\mathrm{cc}-\mathrm{pVTZ})$ & 6.46 & 6.02 & 1.74 & 1.67 \\
\hline PBE & $\mathrm{PW}$ & 6.09 & 5.62 & 1.94 & 1.86 \\
\hline PBE0 & $\mathrm{Ce}(\mathrm{RSC} 97-\mathrm{Tr} .1) / \mathrm{O}\left(6-31 \mathrm{G}^{*}\right)$ & 8.40 & 8.00 & 4.17 & 4.02 \\
\hline PBE0 & Ce(RSC97-Tr.3)/O(cc-pVTZ) & 8.46 & 8.08 & 4.06 & 3.94 \\
\hline B3LYP & $\mathrm{Ce}(\mathrm{RSC} 97-\mathrm{Tr} .1) / \mathrm{O}\left(6-31 \mathrm{G}^{*}\right)$ & 8.00 & 7.59 & 3.51 & 3.38 \\
\hline B3LYP & $\mathrm{Ce}(\mathrm{RSC} 97-\mathrm{Tr} .3) / \mathrm{O}(\mathrm{cc}-\mathrm{pVTZ})$ & 8.06 & 7.65 & 3.42 & 3.30 \\
\hline $\mathrm{LDA}+\mathrm{U}$ & PW & 5.63 & 5.14 & 2.66 & 2.45 \\
\hline $\mathrm{PBE}+\mathrm{U}$ & PW & 5.67 & 5.23 & 2.44 & 2.28 \\
\hline Experimental & $\cdots$ & $5.5-7.0$ & & $2.5-3.5$ & \\
\hline
\end{tabular}


TABLE IV. Cell parameter, $a_{0}$, bulk modulus, $\mathrm{B}$, and elastic constant, $\mathrm{C}_{44}$, of $\mathrm{CeO}_{2}$, using the functionals LDA, PBE, PBE0, and B3LYP. We used the basis sets Ce(RSC97-Tr.1)/O(8-51G), Ce(RSC97-Tr.1)/O(6-31G*), $\mathrm{Ce}(\mathrm{RSC} 97-\mathrm{Tr} .1) / \mathrm{O}(\mathrm{cc}-\mathrm{pVTZ})$, and $\mathrm{Ce}(\mathrm{RSC} 97-\mathrm{Tr} .3) / \mathrm{O}(\mathrm{cc}-\mathrm{pVTZ})$. Results using a PW basis are shown for comparison.

\begin{tabular}{|c|c|c|c|c|c|}
\hline Functional & Basis set & $\begin{array}{l}a_{0} \\
(\AA)\end{array}$ & $\begin{array}{c}\mathrm{B} \\
(\mathrm{GPa})\end{array}$ & $\begin{array}{c}\mathrm{C}_{44} \\
(\mathrm{GPa})\end{array}$ & Ref. \\
\hline LDA & $\mathrm{Ce}(\mathrm{RSC} 97-\mathrm{Tr} .1) / \mathrm{O}(8-51 \mathrm{G})$ & 5.3631 & 207 & $\cdots$ & This work \\
\hline LDA & $\mathrm{Ce}(\mathrm{RSC} 97-\mathrm{Tr} .1) / \mathrm{O}(6-31 \mathrm{G})$ & 5.3623 & 208 & 72 & This work \\
\hline LDA & $\mathrm{Ce}(\mathrm{RSC} 97-\mathrm{Tr} .1) / \mathrm{O}\left(6-31 \mathrm{G}^{*}\right)$ & 5.3510 & 210 & 66 & This work \\
\hline LDA & $\mathrm{Ce}(\mathrm{RSC} 97-\mathrm{Tr} .1) / \mathrm{O}(\mathrm{cc}-\mathrm{pVTZ})$ & 5.3505 & 216 & 86 & This work \\
\hline LDA & Ce(RSC97-Tr.3)/O(cc-pVTZ) & 5.3711 & 220 & 66 & This work \\
\hline LDA & $\mathrm{PW}$ & 5.3667 & 204 & 72 & This work \\
\hline LDA & $\mathrm{Ce}($ Large-core $) / \mathrm{O}\left(8411-1 \mathrm{~d}^{*}\right)$ & 5.399 & 232 & $\cdots$ & 27 \\
\hline LDA & $\mathrm{Ce}($ Small-core $) / \mathrm{O}\left(6-31 \mathrm{G}^{*}\right)$ & 5.360 & 204 & $\cdots$ & 18 \\
\hline PBE & $\mathrm{Ce}(\mathrm{RSC} 97-\mathrm{Tr} .1) / \mathrm{O}\left(6-31 \mathrm{G}^{*}\right)$ & 5.4648 & 170 & $\cdots$ & This work \\
\hline PBE & $\mathrm{Ce}(\mathrm{RSC} 97-\mathrm{Tr} .3) / \mathrm{O}(\mathrm{cc}-\mathrm{pVTZ})$ & 5.4804 & 179 & $\cdots$ & This work \\
\hline PBE & $\mathrm{PW}$ & 5.4674 & 174 & $\cdots$ & This work \\
\hline PBE & $\mathrm{Ce}($ Large-core $) / \mathrm{O}\left(8411-1 \mathrm{~d}^{*}\right)$ & 5.478 & 205 & $\cdots$ & 27 \\
\hline PBE & $\mathrm{Ce}($ Small-core $) / \mathrm{O}\left(6-31 \mathrm{G}^{*}\right)$ & 5.468 & 171 & $\cdots$ & 18 \\
\hline PBE0 & Ce(RSC97-Tr.3)/O(cc-pVTZ) & 5.4110 & 220 & $\cdots$ & This work \\
\hline PBE0 & $\mathrm{PW}$ & 5.39 & $\cdots$ & $\cdots$ & 13 \\
\hline PBE0 & $\mathrm{Ce}($ Large-core $) / \mathrm{O}\left(8411-1 \mathrm{~d}^{*}\right)$ & 5.439 & 224 & $\cdots$ & 27 \\
\hline HSE & $\mathrm{Ce}($ Small-core $) / \mathrm{O}\left(6-31 \mathrm{G}^{*}\right)$ & 5.408 & 206 & $\cdots$ & 18 \\
\hline B3LYP & $\mathrm{Ce}(\mathrm{RSC} 97-\operatorname{Tr} .3) / \mathrm{O}(\mathrm{cc}-\mathrm{pVTZ})$ & 5.4753 & 199 & $\cdots$ & This work \\
\hline B3LYP & $\mathrm{Ce}($ Large-core $) / \mathrm{O}\left(8411-1 \mathrm{~d}^{*}\right)$ & 5.429 & 211 & $\cdots$ & 27 \\
\hline $\mathrm{LDA}+\mathrm{U}(\mathrm{U}=6 \mathrm{eV})$ & PW & 5.4045 & $\cdots$ & $\cdots$ & This work \\
\hline Expt. $^{\mathrm{a}}$ & $\cdots$ & 5.391 & $\cdots$ & $\cdots$ & $\cdots$ \\
\hline Expt. & $\cdots$ & $\cdots$ & $220(9)$ & $\cdots$ & 54 \\
\hline Expt. & $\cdots$ & $\cdots$ & 204 & 60 & 55 \\
\hline
\end{tabular}

${ }^{\mathrm{a}}$ Extrapolated to $0 \mathrm{~K}$ from the data in Ref. 56.

The hybrid results (PBE0 and B3LYP) appear to yield O $2 p \rightarrow$ Ce $5 d$ gaps much larger than most experiments. The smaller band gap of B3LYP compared to PBE0 is expected and is due to the smaller fraction of HF exchange included. Our calculated values for hybrid gaps are larger than those calculated by Baranek et al. ${ }^{27}$ We should note that those authors used what we in this work refer to as a largecore basis set for ceria. Our PBE0 band gaps are consistent with those from the PBE0 PW calculations by Da Silva et $a l .,{ }^{13}$ but a little larger than those obtained from the HSE functional in the work by Hay et al. ${ }^{18}$ and Da Silva et al. ${ }^{13}$ This behavior is expected and is related to the screening parameter introduced in the HSE functional. Paier et al. ${ }^{53}$ showed that PBE0 band gaps were almost uniformly shifted by $0.8 \mathrm{eV}$ relative to the HSE for large band gap semiconductors.

Our B3LYP results appear to indicate a behavior quite different from the behavior reported by Muscat et $a .^{23}$ In their study the experimental band gaps of a large number of metal oxides were well reproduced by the B3LYP functional. However, we should keep in mind that there is a large spread in the experimental data.

The $\mathrm{O} 2 p \rightarrow \mathrm{Ce} 4 f$ gaps from the B3LYP calculations are more in line with experimental measurements than the PBE0, LDA, and PBE values.
All in all, the $\mathrm{LDA}+\mathrm{U}$ and $\mathrm{PBE}+\mathrm{U}$ functionals seem to yield the most consistent description of the band gaps followed by the hybrid B3LYP, while PBE0, LDA, and PBE perform worst. However, due to the uncertainties in the experimental measurements, none of the functionals can be ruled out based on this analysis alone.

\section{E. Cell parameters and elastic constants of $\mathrm{CeO}_{2}$ 1. Comparison of the oxygen basis set}

We have calculated the cell parameter, bulk modulus, and the $\mathrm{C}_{44}$ elastic constant of $\mathrm{CeO}_{2}$ using the LDA functional and the $\mathrm{Ce}(\mathrm{RSC} 97-\mathrm{Tr} .1)$ cerium basis set in combination with three oxygen basis sets $\mathrm{O}(8-51 \mathrm{G}), \mathrm{O}(6-31 \mathrm{G})$, $\mathrm{O}\left(6-31 \mathrm{G}^{*}\right)$, and $\mathrm{O}(\mathrm{cc}-\mathrm{pVTZ})$. Here we are again interested in comparing the quality of the basis sets by comparing them to the PW calculation. The results are shown in Table IV. Gennard et al. ${ }^{57}$ showed in their HF study of ceria that the description of the elastic constants $\mathrm{C}_{11}$ and $\mathrm{C}_{12}$ are little affected by oxygen $d$-orbital polarization functions in the oxygen basis set, suggesting that the bulk modulus should be little affected as well. They observed a large effect from the oxygen $d$-polarization function on the $\mathrm{C}_{44}$ elastic constant. The observation in our work is that the bulk modulus using LDA is quite insensitive to the oxygen $d$-orbital polarization 
TABLE V. Cell parameters, $a_{0}, c_{0}$ and internal parameters $u_{\mathrm{Ce}}, u_{\mathrm{O}}$ of $\mathrm{Ce}_{2} \mathrm{O}_{3}$ using the functionals LDA, PBE, PBE0, and B3LYP. We used the Ce(RSC97-Tr.1)/O(6-31G*) and Ce(RSC97-Tr.3)/O(cc-pVTZ). PW results are also shown for comparison.

\begin{tabular}{|c|c|c|c|c|c|}
\hline Functional & Basis set & $\begin{array}{c}a_{0} \\
(\AA)\end{array}$ & $\begin{array}{c}c_{0} \\
(\AA)\end{array}$ & $u_{\mathrm{Ce}}$ & $u_{\mathrm{O}}$ \\
\hline LDA & $\mathrm{Ce}(\mathrm{RSC} 97-\mathrm{Tr} .1) / \mathrm{O}(6-31 \mathrm{G})$ & 3.8192 & 5.8434 & 0.2432 & 0.6466 \\
\hline LDA & $\mathrm{Ce}(\mathrm{RSC} 97-\mathrm{Tr} .1) / \mathrm{O}\left(6-31 \mathrm{G}^{*}\right)$ & 3.8115 & 5.8323 & 0.2433 & 0.6466 \\
\hline LDA & $\mathrm{Ce}(\mathrm{RSC} 97-\operatorname{Tr} .3) / \mathrm{O}(\mathrm{cc}-\mathrm{pVTZ})$ & 3.7969 & 5.7704 & 0.2369 & 0.6460 \\
\hline LDA & $\mathrm{PW}$ & 3.7660 & 5.8722 & 0.2428 & 0.6413 \\
\hline PBE & Ce(RSC97-Tr.1)/O(6-31G*) & 3.8782 & 5.9687 & 0.2405 & 0.6491 \\
\hline PBE & Ce(RSC97-Tr.3)/O(cc-pVTZ) & 3.8805 & 5.9645 & 0.2400 & 0.6488 \\
\hline PBE & PW & 3.8294 & 6.0717 & 0.2460 & 0.6420 \\
\hline PBE0 & $\mathrm{Ce}(\mathrm{RSC} 97-\mathrm{Tr} .1) / \mathrm{O}\left(6-31 \mathrm{G}^{*}\right)$ & 3.8667 & 6.0767 & 0.2460 & 0.6462 \\
\hline PBE0 & $\mathrm{Ce}(\mathrm{RSC} 97-\mathrm{Tr} .3) / \mathrm{O}(\mathrm{cc}-\mathrm{pVTZ})$ & 3.8711 & 6.0739 & 0.2452 & 0.6463 \\
\hline $\mathrm{PBEO}^{\mathrm{a}}$ & PW & 3.87 & 6.06 & 0.2460 & 0.6459 \\
\hline B3LYP & $\mathrm{Ce}(\mathrm{RSC} 97-\mathrm{Tr} .1) / \mathrm{O}\left(6-31 \mathrm{G}^{*}\right)$ & 3.8945 & 6.2055 & 0.2473 & 0.6459 \\
\hline B3LYP & $\mathrm{Ce}(\mathrm{RSC} 97-\operatorname{Tr} .3) / \mathrm{O}(\mathrm{cc}-\mathrm{pVTZ})$ & 3.8970 & 6.1944 & 0.2466 & 0.6458 \\
\hline $\mathrm{LDA}+\mathrm{U}(\mathrm{U}=6 \mathrm{eV})$ & PW & 3.8557 & 6.0429 & 0.2464 & 0.6432 \\
\hline $\mathrm{LDA}+\mathrm{U}(\mathrm{U}=5.3 \mathrm{eV})^{\mathrm{a}}$ & PW & 3.87 & 5.93 & 0.2441 & 0.6463 \\
\hline Expt. $(@ 4.2 \mathrm{~K})^{\mathrm{b}}$ & $\cdots$ & $\cdots$ & $\cdots$ & $0.251(3)$ & $0.648(3)$ \\
\hline Expt. $(@ 300 \mathrm{~K})^{\mathrm{b}}$ & $\cdots$ & $\cdots$ & $\cdots$ & $0.248(3)$ & $0.648(2)$ \\
\hline Expt. $(@ 300 \mathrm{~K})^{\mathrm{c}}$ & $\cdots$ & $3.891(1)$ & $6.059(1)$ & $0.24543(3)$ & $0.6471(5)$ \\
\hline
\end{tabular}

Reference 13.

${ }^{\mathrm{b}}$ Reference 59.

${ }^{\mathrm{c}}$ Reference 61 .

function just as in the HF study of Gennard et $a l .{ }^{57}$ Moreover, the effect on the $\mathrm{C}_{44}$ elastic constant is not as pronounced in our work using the small-core basis sets and the LDA functional.

\section{Comparison of the functionals}

The choice of functional has a large impact on the resulting cell parameter. Results from calculations using the LDA, PBE, PBE0, and B3LYP functionals are included in Table IV. The cell parameters obtained using PBE and B3LYP are too large compared to the experimental room temperature value. Since the values obtained in a DFT calculation of this type do not take any thermal effects into account a more correct comparison should use a $0 \mathrm{~K}$ extrapolated value. Extrapolating data of Rossignol et al. ${ }^{56}$ yields a cell parameter of about $5.39 \AA$ for ceria. In this case the disagreement between the PBE and B3LYP calculations and experiment becomes even larger. The best fit is obtained using PBE0 while LDA gives too small a cell parameter for ceria. This conclusion agrees with previous PW calculations by Da Silva et al. using LDA, PBE, and PBE0 and with the work of Hay et al., where they used localized atomic basis sets and the functionals LDA, PBE, and HSE.

Note that Baranek et al. ${ }^{27}$ and Voloshina et al. ${ }^{58}$ have done similar comparisons. However, they used large-core ECPs, which we have now shown to be insufficient. Their results are nevertheless included in Table IV for comparison.

\section{F. Cell parameters of $\mathrm{Ce}_{2} \mathrm{O}_{3}$}

We also calculated the cell parameters of $\mathrm{Ce}_{2} \mathrm{O}_{3}$ in the ferromagnetic configuration using the functionals LDA, PBE, PBE0, and B3LYP; see Table V. Here we used the $\mathrm{Ce}(\mathrm{RSC} 97-\mathrm{Tr} .1) / \mathrm{O}\left(6-31 \mathrm{G}^{*}\right)$ and $\mathrm{Ce}(\mathrm{RSC} 97-\mathrm{Tr} .3) / \mathrm{O}(\mathrm{cc}-$ pVTZ) basis sets. PW results using the functionals LDA, $\mathrm{PBE}$, and $\mathrm{LDA}+\mathrm{U}(\mathrm{U}=6 \mathrm{eV})$ are included for comparison. With the basis set $\mathrm{Ce}(\mathrm{RSC} 97-\mathrm{Tr} .1) / \mathrm{O}\left(6-31 \mathrm{G}^{*}\right)$, Hay et al. ${ }^{18}$ found the cell parameters, $a_{0}$ (and $c_{0}$ ), of $\mathrm{Ce}_{2} \mathrm{O}_{3}$ using LDA and PBE to be $3.776 \AA(5.859 \AA)$ and $3.877 \AA(5.978 \AA)$, respectively. The discrepancy between the LDA results obtained in this work and in the work by Hay et al. are discussed in a footnote. ${ }^{60}$

The results obtained using LDA and PBE compare reasonable well (PBE0 even better) to the PW reference. [The results from the PW calculations in this work are in good agreement with previous published results for PW calculations of $\mathrm{Ce}_{2} \mathrm{O}_{3}$ in the Ferromagnetic (FM) state. ${ }^{9,11,13}$ ]

The direct comparison of the calculated cell parameters to experimental cell parameters are more difficult in the case of $\mathrm{Ce}_{2} \mathrm{O}_{3}$, where we have four parameters to consider and again, we must bear in mind that direct comparison should not be made to the room temperature experimental values.

The functionals PBE, PBE0, and $\mathrm{LDA}+\mathrm{U}$ all appear to yield $\mathrm{Ce}_{2} \mathrm{O}_{3}$ cell parameters in good agreement with experiment. LDA underestimates the $c_{0}$ parameter using both the PW basis set and the atomic basis sets. B3LYP on the other hand overestimates the $c_{0}$ parameter. 


\section{SUMMARY}

We conclude that a reliable description of the electronic structure of both $\mathrm{CeO}_{2}$ and $\mathrm{Ce}_{2} \mathrm{O}_{3}$ depends strongly on the description of the core region of cerium. We have shown that the PAW method fails to describe the electronic structure of $\mathrm{CeO}_{2}$ when we use the soft potential for cerium, instead of the standard potential. Similarly, the 46 electron large-core atomic basis sets fail to describe the electronic structure of $\mathrm{Ce}_{2} \mathrm{O}_{3}$, and we instead need to use the 28 electron small-core basis sets.

Thus we conclude that a consistent description of the electronic properties of both $\mathrm{CeO}_{2}$ and $\mathrm{Ce}_{2} \mathrm{O}_{3}$ is obtained by using a small-core basis set for cerium with carefully adjusted diffuse functions or by using a hard potential in the PAW method. We observe large changes by including $d$-orbital polarization functions on the oxygen basis sets. However, we cannot rule out the possibility that these become important for systems with defects, impurities, surfaces, etc., and it is also appealing to treat both oxygen and cerium with comparable quality, which we have achieved in our Ce(RSC97-Tr.3)/O(cc-pVTZ) basis set. Qualitatively, both hybrid functionals PBE0 and B3LYP give a description of $\mathrm{CeO}_{2}$ and $\mathrm{Ce}_{2} \mathrm{O}_{3}$, which is consistent with experiment, at least if we assume the largest plausible levels of experimental uncertainty. However, while both are reasonably good, neither is as good as $\mathrm{LDA}+\mathrm{U}$ or $\mathrm{PBE}+\mathrm{U}$ with their material specific adjustable $\mathrm{U}$ parameters. Comparing the two hybrid functionals, PBE0 performs better than B3LYP when it comes to structural parameters, while B3LYP performs better for the electronic properties.

\section{ACKNOWLEDGMENTS}

We would like to acknowledge the Swedish Foundation for International Cooperation in Research and Higher Education (STINT) and the Swedish Research Council (VR) for financial support and the Swedish Infrastructure for Computing (SNIC) for computing resources. The authors would like to thank Professor Richard Martin, Professor Beate Paulus, and Dr. Elena Voloshina for valuable discussions. D.M.R. also acknowledges the support from the Grant-in-Aid for Creative Scientific Research (Grant No. 16GS0205) from the Japanese Ministry of Education, Culture, Sports, Science, and Technology and C.W.M.C. acknowledges the support from the J. Gust. Richert stiftelse.

${ }^{1}$ F. Marabelli and P. Wachter, Phys. Rev. B 36, 1238 (1987).

${ }^{2}$ D. R. Mullins, S. H. Overbury, and D. R. Huntley, Surf. Sci. 409, 307 (1998).

${ }^{3}$ W. Chengyun, Z. Weiya, and Q. Yitai, Mater. Sci. Eng., B 94, 170 (2002).

${ }^{4}$ E. Wuilloud, B. Delley, W.-D. Schneider, and Y. Baer, Phys. Rev. Lett. 53, 202 (1984)

${ }^{5}$ A. Pfau and K. D. Schierbaum, Surf. Sci. 321, 71 (1994).

${ }^{6}$ C. Chai, S. Yang, Z. Liu, M. Liao, and N. Chen, Chin. Sci. Bull. 48, 1198 (2003).

${ }^{7}$ C. W. M. Castleton, J. Kullgren, and K. Hermansson, J. Chem. Phys. 127, 244704 (2007)

${ }^{8}$ A. V. Prokofiev, A. I. Shelykh, and B. T. Malekh, J. Alloys Compd. 242, 41 (1996).

${ }^{9}$ D. A. Andersson, S. I. Simak, B. Johansson, I. A. Abrikosov, and N. V. Skorodumova, Phys. Rev. B 75, 035109 (2007).

${ }^{10}$ S. Fabris, S. de Gironcoli, S. Baroni, G. Vicario, and G. Balducci, Phys.
Rev. B 71, 041102 (2005).

${ }^{11}$ C. Loschen, J. Carrasco, K. M. Neyman, and F. Illas, Phys. Rev. B 75, 035115 (2007).

${ }^{12}$ Y. Jiang, J. B. Adams, and M. van Schilfgaarde, J. Chem. Phys. 123, 064701 (2005)

${ }^{13}$ J. L. F. Da Silva, M. V. Ganduglia-Pirovano, J. Sauer, V. Bayer, and G. Kresse, Phys. Rev. B 75, 045121 (2007).

${ }^{14}$ A. Becke, J. Chem. Phys. 98, 5648 (1993).

${ }^{15}$ C. Lee, W. Yang, and R. Parr, Phys. Rev. B 37, 785 (1988).

${ }^{16}$ S. Vosko, L. Wilk, and M. Nusair, Can. J. Phys. 58, 1200 (1980).

${ }^{17}$ P. Stephens, F. Devlin, C. Chabalowski, and M. Frisch, J. Phys. Chem. 98, 11623 (1994)

${ }^{18}$ P. J. Hay, R. L. Martin, J. Uddin, and G. E. Scuseria, J. Chem. Phys. 125, 034712 (2006)

${ }^{19}$ J. P. Perdew, K. Burke, and M. Ernzerhof, Phys. Rev. Lett. 77, 3865 (1996).

${ }^{20}$ J. Tao, J. P. Perdew, V. N. Staroverov, and G. E. Scuseria, Phys. Rev. Lett. 91, 146401 (2003).

${ }^{21}$ J. Heyd, G. E. Scuseria, and M. Ernzerhof, J. Chem. Phys. 118, 8207 (2003).

${ }^{22}$ T. Bredow and A. R. Gerson, Phys. Rev. B 61, 5194 (2000).

${ }^{23}$ J. Muscat, A. Wander, and N. M. Harrison, Chem. Phys. Lett. 342, 397 (2001).

${ }^{24}$ V. Vetere, C. Adamo, and P. Maldivi, Chem. Phys. Lett. 325, 99 (2000).

${ }^{25}$ C. Müller, C. Freysoldt, M. Baudin, and K. Hermansson, Chem. Phys. 318, 180 (2005).

${ }^{26}$ B. Herschend, M. Baudin, and K. Hermansson, Chem. Phys. 328, 345 (2006).

${ }^{27}$ P. Baranek, L. Gauthier, and M. Marrony, AIP Conf. Proc. 963, 367, (2007).

${ }^{28}$ M. Dolg, H. Stoll, and H. Preuss, J. Chem. Phys. 90, 1730 (1989).

${ }^{29}$ Excitation from the $4 f^{1} 5 d^{1} 6 s^{21} \mathrm{G}$ ground state to the $5 d^{2} 6 s^{23} \mathrm{~F}, 5 d^{1} 6 s^{35} \mathrm{~F}$, $5 d^{45} \mathrm{D}, 4 f^{1} 5 d^{2} 6 s^{15} \mathrm{I}, 4 f^{1} 5 d^{35} \mathrm{I}, 4 f^{2} 6 s^{23} \mathrm{H}, 4 f^{2} 5 d^{1} 6 s^{15} \mathrm{~K}$, and $4 f^{2} 5 d^{25} \mathrm{~L}$ final states were considered.

${ }^{30}$ X. Cao and M. Dolg, J. Chem. Phys. 115, 7348 (2001).

${ }^{31}$ X. Cao and M. Dolg, J. Mol. Struct.: THEOCHEM 581, 139 (2002).

${ }^{32}$ D. Feller, J. Comput. Chem. 17, 1571 (1996).

${ }^{33}$ M. Dolg, P. Fulde, W. Kuchle, C.-S. Neumann, and H. Stoll, J. Chem. Phys. 94, 3011 (1991).

${ }^{34}$ M. Huelsen, A. Weigand, and M. Dolg, Theor. Chem. Acc. 122, 23 (2009).

${ }^{35}$ T. R. Cundari and W. J. Stevens, J. Chem. Phys. 98, 5555 (1993).

${ }^{36}$ W. J. Stevens, M. Krauss, H. Basch, and P. G. Jasien, Can. J. Chem. 70, 612 (1992)

${ }^{37}$ V. I. Anisimov and O. Gunnarsson, Phys. Rev. B 43, 7570 (1991).

${ }^{38}$ M. Ernzerhof and G. E. Scuseria, J. Chem. Phys. 110, 5029 (1999).

${ }^{39}$ C. Adamo and V. Barone, J. Chem. Phys. 110, 6158 (1999).

${ }^{40}$ M. J. Frisch, G. W. Trucks, H. B. Schlegel et al., GaUSSIAN 03, Revision C.02, Gaussian, Inc., Wallingford, CT, 2004.

${ }^{41}$ R. Dovesi, V. Saunders, C. Roetti, R. Orlando, C. M. Zicovich-Wilson, F. Pascale, B. Civalleri, K. Doll, N. M. Harrison, I. Bush, Ph. D'Arco, and M. Llunell, CRYSTAL06 User's Manual (2006).

${ }^{42}$ G. Kresse and J. Hafner, Phys. Rev. B 49, 14251 (1994).

${ }^{43}$ G. Kresse and J. Furthmüller, Comput. Mater. Sci. 6, 15 (1996).

${ }^{44}$ H. J. Monkhorst and J. D. Pack, Phys. Rev. B 13, 5188 (1976).

${ }^{45}$ M. Causa, R. Dovesi, C. Pisani, and C. Roetti, Phys. Rev. B 33, 1308 (1986)

${ }^{46}$ W. Hehre, R. Ditchfield, and J. Pople, J. Chem. Phys. 56, 2257 (1972).

${ }^{47}$ P. Hariharan and J. Pople, Theor. Chim. Acta 28, 213 (1973).

${ }^{48}$ J. T. H. Dunning, J. Chem. Phys. 90, 1007 (1989).

${ }^{49}$ P. E. Blöchl, Phys. Rev. B 50, 17953 (1994).

${ }^{50}$ X. Gonze, P. Käckell, and M. Scheffler, Phys. Rev. B 41, 12264 (1990).

${ }^{51}$ G. Kresse, P. Blaha, J. L. F. D. Silva, and M. V. Ganduglia-Pirovano, Phys. Rev. B 72, 237101 (2005).

${ }^{52}$ S. Fabris, S. de Gironcoli, S. Baroni, G. Vicario, and G. Balducci, Phys. Rev. B 72, 237102 (2005).

${ }^{53}$ J. Paier, M. Marsman, K. Hummer, G. Kresse, I. C. Gerber, and J. G. Angyan, J. Chem. Phys. 124, 154709 (2006).

${ }^{54}$ L. Gerward, J. S. Olsen, L. Petit, G. Vaitheeswaran, V. Kanchana, and A. Svane, J. Alloys Compd. 400, 56 (2005).

${ }^{55}$ A. Nakajima, A. Yoshihara, and M. Ishigame, Phys. Rev. B 50, 13297 (1994)

${ }^{56}$ S. Rossignol, F. Gerard, D. Mesnard, C. Kappenstein, and D. Duprez, J. Mater. Chem. 13, 3017 (2003) 
${ }^{57}$ S. Gennard, F. Cora, C. Richard, and A. Catlow, J. Phys. Chem. B 103, 10158 (1999).

${ }^{58}$ E. Voloshina and B. Paulus, J. Chem. Phys. 124, 234711 (2006).

${ }^{59}$ H. Pinto, M. H. Mintz, M. Melamud, and H. Shaked, Phys. Lett. 88A, 81 (1982).

${ }^{60} \mathrm{P}$. J. Hay et al. (Ref. 18) include a $g$-projector in the ECP, and we do not, but the exclusion of this term is not expected to have a large effect. The PBE results are in good agreement with those we obtained using their basis set (without the $g$-projector), but there is a discrepancy in the LDA results which we have not been able to explain. For example changing from the LDA parametrization of von Barth-Hedin (Ref. 62), used routinely in our calculations, to Dirac-Slater exchange (Ref. 63) and Vosko-Wilk-Nusair correlation (Ref. 16) only give a change in the lattice parameters of $\sim 0.01 \AA$. In the original paper by Vosko et al. several parametrizations are presented, and the CRYSTAL and GAUSSIAN codes use different sets by default. The default setting in GAUSSIAN is not available in CRYSTAL, but on the basis of the other tests this is unlikely to explain the discrepancy, so our test is not complete.

${ }^{61}$ H. Bärnighausen and G. Schiller, J. Less- Common Met. 110, 385 (1985).

${ }^{62}$ U. von Barth and L. Hedin, J. Phys. C 5, 1629 (1972).

${ }^{63}$ P. A. M. Dirac, Proc. Cambridge Philos. Soc. 26, 376 (1930). 